\title{
Data Intensive Computing using SoSE Architecture for Healthcare Information System
}

\author{
Hanumantha $\mathrm{K} \mathrm{N}^{1}$, Sunitha $\mathrm{S}^{2}$ \\ M.Tech, IV Sem, Dept of CSE, RYMEC Ballari, VTU University ${ }^{1}$ \\ Assistant Prof, Dept of CSE, RYMEC Ballari, VTU University²
}

\begin{abstract}
The design of tending data system is a vital framework. The design implements the last technologies of cloud computing and large knowledge in parallel and distributed computing manors. The design theme is planned in SoSE-based systems engineering paradigm. The implementation of such design for tending to process is given via multiple layers of huge knowledge implementations. This study may be a primary work that provides basic insight into the future intelligent system of HIS systems.
\end{abstract}

Index Terms: Healthcare Information Systems (HIS), Big Data, Cloud Computing, Healthcare Services, System Architecture, The System of Systems, Sose, Data Intensive Computing, HPC, Parallel Computing.

\section{INTRODUCTION}

The construct of large-scale computing (massive computing) isn't new coin. The term has been used throughout the complete domain of computations, especially parallel computing, supercomputing, and high-performance computing. It always refers to the scale of central processing unit computing capability like petascale computing exascale computing. It implicitly stands for how several processors used and the way to hurry up computations with high quantifiability. However, the term of large-scale computing is additionally adopted in information intensive computing. As an example, the conception of large-scale is applied to not solely what number CPUsbeing used however additionally, however, massive the datasets concerned in a single computation. It additionally includes multiple information storages participated in an exceedingly single or sizable amount of computing tasks to be accomplished victimization of computing capability and resources.

In general, a large-scale information intensive computing has 5 elements of sub-systems as shown in below figure.

1. High performance of computing systems equipment

2. $\quad$ High-performance code completed calculation task by wants

3. High performance of network system and corresponding of facilities

4. High-performance information management system

5. Computational Expert is an HR system

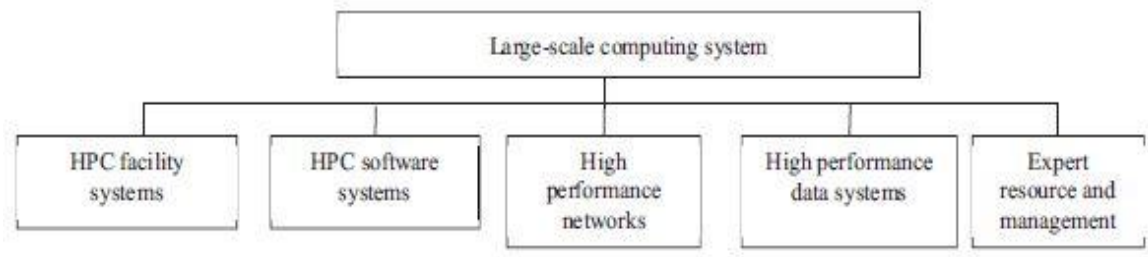

Fig1: Traditional Hierarchical Framework of Large-Scale

\section{SYSTEM OF SYSTEMS}

In order to aggregate all the all the HIS system components to work together, one needs to well understand each function of the system components (as we described above), and their inter-cooperative and interactions.

The system is a set of interacting or interdependent components (elements) forming an integrated whole.

The system can be applied to different application domains. If the term is applied to form a set of engineering components, we usually call it an engineering system. If the term is applied to a biological entity, we refer it as a biological system, as such human body. In science and engineering, people often extract common abstract properties 
(characteristics) among various systems in terms of their matter, organization, behaviors, and principles which are independent of the specific domain, substance, type, or temporal scales of existence.

A system is composed of many system components such as out HIS subsystems or second layer sub-systems. These components can be basic elements or can be subsystems or componential systems which consist of their own elements or systems. The componential systems are called subsystems, while their parent becomes a super system. The componential elements or systems can be either homogeneous or heterogeneous to identify if the elements or subsystems are uniform or well-mixed.All the nature systems and most of the engineering systems contain heterogeneous elements. These systems' elements are numerous types in terms of their attributes, characteristics, behaviors, functions, and goals.

One cannot use systems classification to spot whether the system is natural or synthetic or engineered. As an example, as we tend to all noted, all the nature systems have subsystems, that even have their own subsystems. It forms Associate in the nursing infinite hierarchical division, as long as the matter may be classified into smaller entities. Supported this paradigm, an engineering system may be infinite classified into smaller matters, the same as biological Classification. Therefore, the identification of nature or engineering systems relies on its self- and natural characteristics, behaviors, and goals. A natural system' elements, for example, have their own naturally-grew, independent behaviors, functions, rules, and goals. If their attributes and functions are damaged, they can utilize the open system boundaries

To self-adapt environmental changes, self-adjust their

Functions, or self-treat their damages, as well as self-control and govern their functions, and rules in order to sustain their survival until the life-cycle ended.However, many engineering systems do not have such properties and intelligent behaviors. Therefore, we believe natural systems are superior, complex and intelligent, even though look very simple. The human's great efforts are indented to a develop engineering systems approach to the natural systems. That is the system engineering final destinations, although it has a long way to go. That opinion implies the difference between the traditional systems engineering approach vs. today systems engineering methodology that is information-netcentric,intelligence-based, open systems architecting and integration. That is the theme of the system of systems engineering (SoSE). The concepts and principles inSoSE will be directly applied to our planned integrated HIS super systems as we tend to represented.

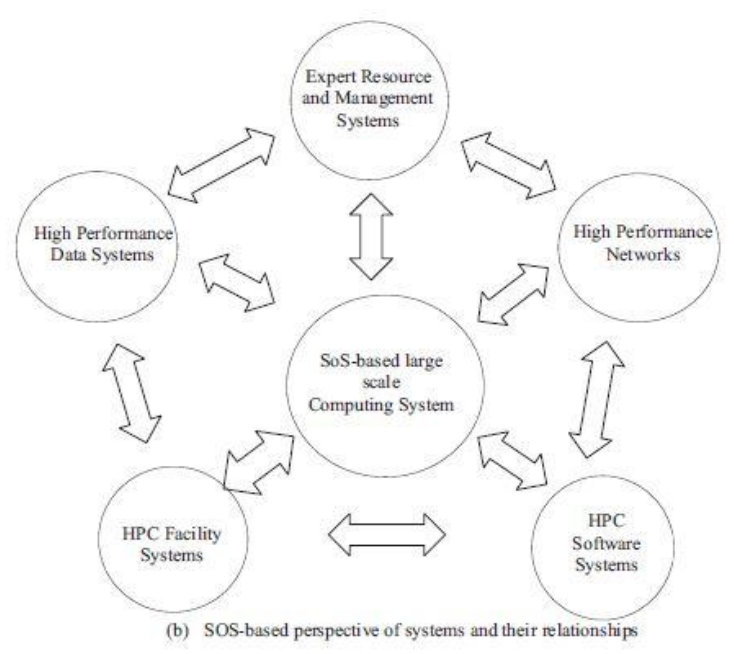

\section{SOSE BASED BIG DATA ON HIS}

\subsection{Hardware architecture and design}

As typical design of aid, the massive information processing system is sketched in Figure a pair of. In general, the design style of a computing system has 2 approaches. One is computation oriented design, and also the different may be a distributed architecture. Centralized process system with information exchange speeds features shared memory, relatively stable in technical operation, moreover as straightforward to manage and maintain. Its obvious disadvantage is computational capability isn't robust to the value, poor scalability, and lack of reading of the updated computing resource. A distributed processing system is exactly to form up for the shortcomings of the centralized computing system. The system will integrate several PC processors and resources together to create a robust distributed-memory system. The system will simply expand to additional servers and easily composed of multi-core processor systems. 
Traditional computing systems cannot meet huge data processing. The mixing of large-scale distributed computing resources for single computational task results in the construct of grid computing, however just for intensive CPUbased computations in the world. On the opposite hand,industrials fairly often to wear down a great deal of distributed information by additionally utilizing multiple computing resources. Supported their business profits, they come up with (1) services-based or utility computing for services charges, (2) technical design settings for distributed information intensive computing, and (3)supportive methodology to integrate resources behind that results in virtual technology. These three aspects of developments generate today's cloud computing with service operational modes (IaaS, PaaS, and $\mathrm{SaaS}$ ), and business model, as well as technical treatment of huge information victimization distributed computing methodology likeHadoop/MapReduce, just like HPC's parallel decomposition of procedure tasks, but distributed-stored information -oriented tasks. The HPC'sparallelism is for computing potency, while big data correspondence is for the capability to processing.

The development of information intensive computing needs to take into account each superior data-file storage system and superior computing system. May computing technology actually used to share the recourses to forming virtual giant data and computing platform. This design requires a superior network crossing multiple platforms or servers. The multiple computational nodes inside HPC scheme is dedicated to mapping and reducing tasks throughout processing management and mining.

The main schema modifications area unit due to 3 subsystems: information storage subsystems, a high-performance computing subsystem, and substantiating info server subsystems. In our information storage subsystems, we adopted disk arrays (Redundant Arrays ofIndependent Disks, RAID) information storage, using different info package to support information storage. We have a tendency to designed 2 information storage system, one is internal however with little information capability and external one which might host giant dataset. Associate in Nursing LAN information converter is employed to attach external systems, the whileInfiniBand switch is employed to link to all or any the interior computational modes inside the HPC scheme. The main objective is to boost potency, strengthen the effectiveness calculations, handling bulk information.Supporting server scheme is employed to participate in different types of calculations, as well as network servers, network information sharing, security entry, network, information delivery, special peripheral information acquisition, data processing, information preprocessing, data visualization, information backup and GPU process.

\subsection{Architecture Configuration of Cloud-based DataProcessing System with Big Data Configuration}

Since the 2 cores in today's massive information technology area unit Hadoop/MapReduce andHDFS. Since MapReduce and Hadoopwerefirst developed and discharged as open sources, they have been wide utilized in several massive information applications. The popularity of victimization has big with the development of further tools like HBase, Pig, Hive, etc. at this time there's no uniform system of technical structures and customary technical standards.Liu recently presents a Hadoop-based computing architecture for processing, shown in below figure.

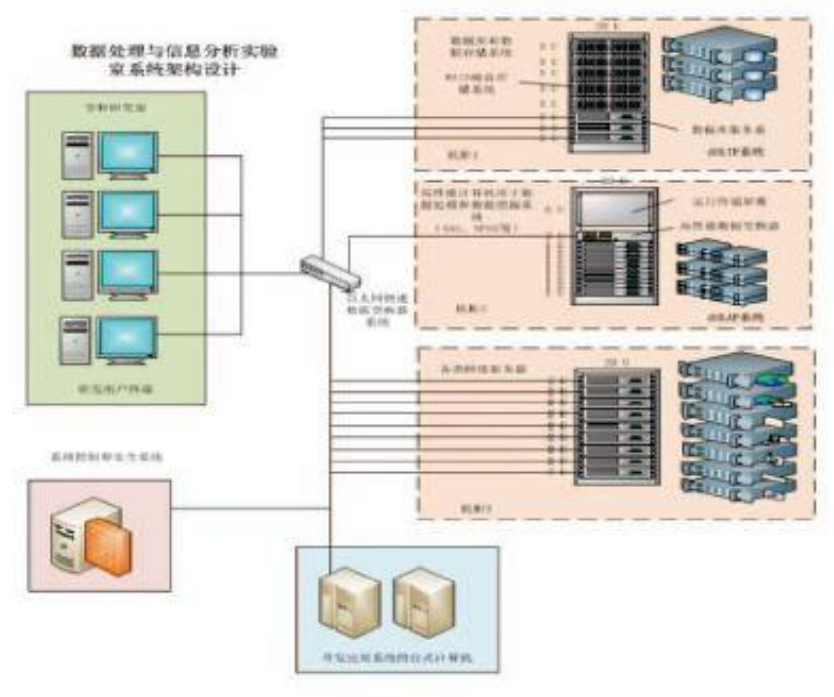

This design has its generality, particularly will be tailored to attention data system. This framework addresses the characteristics of distributed computing systems, sort of a grid ADPS architectures, victimization layers, and specific methodologies mapped to the corresponding application fields. For instance, within the information storage layer, one will use HBase and HCatalog technologies, which facilitate to handle NoSQL information. We tend to address the bottom-up layers in as follows: 
Data Resource Layer: All-time low layer lies on various information resources as well as attention application information, non-public information of patients, families, and residents. This information area unit typically holds on within the enterprise databases. The resources conjointly embody logs data to the systems. The kinds and their data structure area unit terribly various. The info characteristics are unit various in each formatting and arrangement. Some of the data area unit unstructured, semi-structured information. The log information in each text formats and wealthy media form from internet or net returns. A number of this information are hold on centralized whereas others area unit hold on directly distributed classification system (DFS), like formatted log files. Some information is parsed directly byMapReduce programs, whereas others not. However once it comes to take care of ancient attention applications, one will save the info within the primary info, such as historical information from OS, since they are not holding on in HDFS. MapReduce programs area unit required to access them through external API within a the acceptable variety of the info. This approach is neither flexible nor economical, and Hadoop Framework is introduced as an information integration layer. Typical instances of victimization information integration layer like Snoop tool area unit to import information that's hold on in relative databases to Hadoop/MapReduce enabled format for the subsequent handling procedures victimization Hive tool. The other approach is to directly import the info intoHBase, that support exports access information hold on in an exceedingly relational info.

File Storage Layer: File storage layer distributed classification system. The system underlies a number of distributed files at totally different locations via a network affiliation. Through a unified interface, the system provides AN objectlevel file access to application services. File storage application layer deals with the highest defend device sorts and models, the interface protocols, and alternative technical details like storage location. The system provides data backup, fault tolerance, and condition monitoring, security and alternative guarantees reliable document accessible to totally different service management units. Meanwhile, the distributed parallel files are stored in an exceeding information cloud. The info process environment ought to support economical parallel access to huge amounts of huge files. During this overall architecture, file storage layer will handle specific integration by connecting the specified information at remote data storage resources. HDFS could be a core part of the file storage layer.

Data Storage Layer: It's accountable to produce distributed, scalable information storage and management capabilities. Totally different from ancient relative databases, cloud-based information storage doesn't need the full SQL support. The feature of its low price management and fast reads and writes of the info offers 2 tiers of storage technology foundations, HBase and HCatalog. HBase implements a distributed, column-oriented info storage system, while HCatalog is for table-based information management.

The Programming Model Layer: It is reasonable for cryptography MapReduce-based task decomposition, information access, and programming of protocols to access distributed files. It touches the base of algorithms utilized in massive information that depends on targeted applications

Data Analysis Layer: It permits to conduct information analysis with attentions on processing and analysis models, business price, and data extraction, instead of validatory layers. It provides some advanced analysis tools for information analysts.Hadoop Pig and Hive area unit 2 major analysis tools.Pig provides a MapReduce abstracts supported a higher level of data-processing capability, as well as data manipulation language and the operative environment. Hive is that the structured information that may be mapped to table for data analysts, such as providing an entire SQL question, and a question language to throughout MapReduce task execution.

Platform ManagementComponent: One the left-hand-side is that the validatory system. It permits the smooth and safe operation for entire processing.With alternative systems management elements like a cloud, clustering, and watching etc., The PMCcomponent handles system configuration and resources management, watching standing and handling fault, performance standardization, making certain security etc.

In the on top of bestowed four-level framework, according to the addressed HIS environments and applications on surroundings, applicable amendments, and local changes are also created. Every system and subsystems ought to maintain its freelance in self-operation,self-adjusting, and sovereign. The data transferring and communication ought to be designed with open interface configuration with international standards like DICOM, HL7, and other IT standards. Counting on special applications, developers will insert appropriate layers middleware settings. Folks can even develop special interface or plugins for the preparation of outstanding enterprise information analysis and statistics computer code. In order to work the system, some special style and consideration ought to be created in high-performance networking. In procedure model, folks might add a special interface to permit parallel computing throughout data intensive computing. Such combined computational models are terribly helpful in dealing with medical and attention applications, such as image processes like registration, classification, and reconstructions etc. in a clinic or hospital environments. 


\section{CONCLUSION}

There exist large demands of developing and constructing integrated aid data system which includes nowadays largescale computing for giant data. Today's HIS system elements ought to match with aid services. Every HIS scheme is designed to map the corresponding services. The architectures of varied information intensive computing addressed with stress on targeted information quantity, structure, and information storage. The cloud and large information enhanced design are planned during a systems engineering paradigm. The SoSE primarily based design for aid processing is meant via multiple layers with concerns. This study could be a primary work that provides basic insight into the future intelligent system of HIS systems.

\section{REFERENCES}

[1] AnanthGrama, George Karypis, Vipin Kumar, AnshulGupta, Introduction to Parallel Computing (2nd Edition), ISBN 978-0201648652, 2003.

[2] Andrew Ryan, Under the Hood: Hadoop Distributed filesystem reliability with Namenode and Avatarnode, Facebook Blog, 2012.6.

[3] Apache Solr, http://lucene.apache.org/solr/.

[4] Banerjee J., R. I. Baum, and D. K. Hsiao, “Concepts andCapabilities of a Database Computer, " ACM Transactions on Database Systems, Volume 3 Issue 4, 1978.12.

[5] Baum, D.K. Hsiao, "Database Computers-A Step TowardData Utilities,” IEEE Transactions on Computer, Vol. C-25,No. 12, Pages 1254-1259, 1976.12 .

[6] BehroozParhami, “A Highly Parallel Computing System for Information Retrieval,” in AFIPS Proceedings of theFall Joint Computer Conference, Pages 587-595, 1972.

[7] D. R. Brownbridge, L. F. Marshall, and B. Randell, "theNewcastle Connection," Software Practice andExperience12: 1147-1162, 1982.

[8] D. R. McGregor, R. G. Thomson, and W. N. Dawson,"High-Performance Hardware for Database Systems,"Systems for Large Data Bases, Pages 103-116, 1976

[9] D.L. Slotnick, Logic per Track Devices, Advances inComputers, Vol. 10, Academic Press New York, pp. 291-296, 1970.

[10] D.L. Slotnick, Logic per, "Track Devices," Advances inComputers, Vol. 10, Frantz Alt, Ed., Academic Press, NewYork, pp. 291-296, 1970 